\title{
Radiology exam survival guide
}

\section{Sheldon Godinho FCRadD (SA), FRCR \\ Department of Radiology, University of Pretoria}

Who can forget those stressed-out sleepless nights as examinations draw near? Frantic last-minute reading and rehearsing cases. Rumours about examiners and their preferences. Certainly no time for philosophical reflection. And yet so much insight can be gained by applying a little thought to what lies ahead.

The examination procedure endeavours to test major aspects of the training process and therefore consists of written tests, 'long' written cases and 'short' oral cases. MCQs are objective tests, mainly of theoretical (book) knowledge. Formal written exams can also test problem-solving ability. Long cases probe the candidate's approach to more complex diagnoses. Sometimes a rapid-reporting session is included where many spot-diagnoses must be made in a limited time, as in the FRCR.

And ultimately there is the oral examination. This is by far the single most important (and intimidating) test and normally also carries the greatest weight. Everyday diagnostic problems are presented, usually in increasing order of difficulty. A few theoretical questions pertaining to cases shown are possible.

\section{MCQs and written examinations}

Generally speaking, $\mathrm{MCQ}$ s require broad rather than deep knowledge. Reading from different sources is better than attempting to memorise a single book. This way different insights and perspectives are gained and suicidal boredom can be avoided.

Books containing example MCQs are excellent practice. Note the wording of questions. Often a subtle inflexion changes the entire context of a statement and hence the answer, e.g. 'Irregular microcalcifications are diagnostic of invasive breast carcinoma' vs. 'Irregular microcalcifications are a feature of invasive breast carcinoma. The first statement is false and the second is true. Read carefully!

If negative marking is employed, guessing is fatal! Be content knowing the answers to only two out of five sub-questions and mark only these, rather than incorrectly guessing the remaining three, for a grand total of minus one!

Long written questions require more in-depth knowledge but tend to concentrate on 'important' conditions, or sometimes those that are currently topical. Exceptions may occur, however, usually in the form of some recently published obscurity that tickled one examiner's fancy.

Basically only two types of written question can be formulated: pathology-oriented or patient-oriented. A pathology-oriented question will revolve around the imaging features of a particular condition or perhaps a problem approach, often relating to a recent journal publication. It is therefore worthwhile to scour the major journals of the preceding $12-18$ months for suitable articles. If time has run out, at least list the titles and read up on those subjects in a concise text such as the Radiology Review Manual by Dähnert. Second-best and not very entertaining reading, but better than nothing.

Patient-oriented questions sketch a clinical scenario requiring a radiological approach to the problem and expected imaging findings for the differential possibilities.

Concise, point-form answers are logical and easier to mark than Shakespearian essays. The same reasoning applies to legible handwriting vs. standard-fare medical hieroglyphics. Finally, allow equal time per question. Some sort of answer must usually accompany every question, so it is silly to compile an encyclopaedic answer to one question, only to run out of time to answer another.

\section{Long cases}

Two or more films may be supplied for each case along with a short clinical history. The timescale may vary from four cases in 1 hour to six cases in 45 minutes. Findings and conclusions must be handwritten and so obviously, time is a major constraint! Success requires strict adherence to a timetable, allowing equal time per case. No problem if a rotation system is enforced, but another matter entirely if presented with a pile of cases, a blank lightbox and a ticking stopwatch!

A brief description of the patholo- 
gy and major radiological signs should be given, preferably pointform. Following this with a singlesentence summary of the findings is a nice touch before the diagnostic possibilities are tackled, e.g. 'Middle-aged female with invasive chest-wall mass and multiple blastic skeletal foci.' This is also a good approach to oral exams and general reporting.

Sometimes all signs will point to a single condition but often only a differential diagnosis is possible. Even so, one diagnosis is usually more probable than any other and should be listed first. Keep differential lists as short as possible. A telephone book of remote and obscure conditions will not impress! If necessary, add a few notes next to each differential explaining why you consider it more or less likely to be the final diagnosis. Suggest further investigations that could help as well as expected findings on these.

\section{Rapid-reporting sessions}

To the best of my knowledge rapid reporting is not yet employed in any local examinations. The format generally consists of spot diagnoses and 'aunt Mollies', say 30 cases in 30 minutes requiring one-word answers.

\section{The oral examination}

The nail-biting finalé: less pleasant than a romantic dinner for two but not as bad as passing a kidney stone. Two or three 30-minute sessions with two examiners to test your worth as a diagnostician. These are the tools you will require:

1. Knowledge: A basic theoretical foundation must exist and is acquired through the reading of books and articles. The verbal content of meetings and tutorial sessions can be invaluable and is worth recording in a notebook. On seeing something new, take the trouble to read up briefly on that subject. Facts are most easily recalled when coupled to pictures.

2. Experience: Familiarity breeds confidence. Explore the film library with a study-buddy. Seize every opportunity to present cases in meetings and tutorial sessions. Become accustomed to the stress of being under the spotlight. Never be embarrassed to make a mistake - each mistake is a lesson learned and is less likely to be repeated in an exam, or worse, on a patient's report!

3. Exam technique: A brainy candidate who lacks confidence and logical presentation skills is likely worse off than a less academic candidate who has a good, commonsense approach and is not easily rattled. Good technique is nothing more than a thorough, logical system for examining films and approaching different case scenarios.

Good technique backed up by sufficient knowledge, and experience implementing both must meet with success!

The exam session begins with an introduction to the external examiners. Pleasantries over, seat yourself comfortably. Avoid positioning yourself so that one examiner sees only your back.

Most examiners will state at the outset that you are free to handle the films and use the bright light. If not, politely ask before doing so. A pointer is provided. In my opinion though, a better impression is created if an accurate anatomical description of the pathology and its location can be given verbally, as in an X-ray report. Under no circumstances should fingers be used to point out some detail. Aside from appearing agricultural, examiners may take exception to greasy fingerprints on their prized exam films.

The first case is either placed on the lights by the examiner or given to the candidate to put up himself. Should the examiner place a film backwards it is best taken down subtly and replaced correctly, perhaps using the opportunity to read the label information and examine darker bits under the bright light. Heroically flipping the film over will not impress. Also, resist the temptation to catch a glimpse of the diagnosis on the envelope as the examiner extracts his films. When putting films up yourself, check $\mathrm{L}$ and $\mathrm{R}$ markers as well as anatomical side markers, e.g. heart, aorta, liver, gastric bubble. Check the name, sex and patient age on all films, if visible.

During all this you will be given a short, relevant history. Listen and remember! Avoid repeating the history when presenting the case. This is irritating and wastes time. Now you are ready to tackle your first case.

\section{Step 1}

Say absolutely nothing! Take time (say 10 - 20 seconds) to examine the entire film systematically. If you have not already done so, take note of side markers, other annotations, e.g. 'control' or 'supine', film quality, name (race/nationality), age, sex and other information that may be present.

Concentrate on areas of obvious pathology but examine the whole film, including anything possibly hidden behind film clamps. Correct anatomical placement of pathology is 
critical in compiling a sensible differential diagnosis, e.g. anterior, middle or posterior mediastinal masses. Beware of the second pathology! Remember the history!

\section{Step 2}

This next step should be simple and virtually subliminal for most cases shown to a well-prepared candidate: the anomaly is observed and then localised and categorised. This should hold for all straightforward (type I) cases. All that remains is step 3 below, viz presentation.

But not all cases are so straightforward. Hiccups often arise from inevitable gaps in the knowledgeexperience-technique toolkit, especially with more challenging cases. After all, we're only human! I have designated these cases type II when an abnormality is obvious but not the probable cause, and type III when no abnormality is initially apparent.

No cause for panic though. A little thought and insight into case types can help to devise a suitable approach. The basic idea is to gather whatever additional information is needed to reduce a difficult type II or III case to a more 'simple' type I and dispose of it accordingly.

\section{Case-type I: The pathology is clear and diagnosis certain}

There are always cases of this nature included in an exam, taking the form of classic signs, 'aunt Minnies' and the like, often with a typical history. Commonly, a case of this type is shown first to build a little confidence. These are best dispatched quickly and unceremoniously, saving time and allowing more films to be viewed in the remainder of the session.

\section{Case-type II: The pathology is obvious but no definite diagnosis can be made immediately}

Good examples would be a solitary pulmonary nodule or single eroded vertebral pedicle.

Give a full description of all positive and relevant negative findings. Several conditions could likely explain the picture you have described. A differential may be possible but no specific diagnosis as yet. More information is required! Do not be tempted to rattle off a million possibilities in an attempt to sound clever.

In the back of your mind should be the general differential approach, viz. congenital, infective, traumatic, neoplastic, metabolic collagen-vascular, organ-specific, etc. It should be possible, however, to exclude many of these categories based on the history and signs and thereby tailor requests for further clinical information, additional views or further investigations toward those that remain.

Based on further information gathered in this way a definite diagnosis or at least narrow differential ought to be possible.

\section{Case-type III: No pathology is immediately apparent - a 'normal' film}

These films require careful scrutiny as they often conceal abnormalities that should never be missed, e.g. subtle fractures, vague masses, free abdominal air, pulmonary embolism, etc. Once the abnormality is spotted, these become type I or II cases and can be handled accordingly.

\section{Step 3}

Present the case: begin by intro- ducing your patient, the study performed and the views available. It is more professional to use the patient's name whenever possible, e.g. 'This is an $\mathrm{ABC}$ examination of the left/right $\mathrm{PQR}$ of Mr/Mrs XYZ'. Using the name may indicate race without politically incorrect direct reference, and this can be very helpful diagnostically. Virtually exclusive occurrence of sickle-cell disease in black patients and cystic fibrosis in whites are examples.

Approach and presentation will vary according to the case at hand.

\section{Type I case: diagnosis certain}

- Give a brief description of the signs and reach a conclusion quickly.

- Sometimes the diagnosis can be given directly, e.g. 'I see that the patient has sustained a transscaphoid, perilunate fracturedislocation'

- Familiarity with common pathology and a polished presentation technique builds the confidence needed to come directly to the point and proceed quickly.

- If you are correct, over to the next case. In the event that you have been too hasty and over-confident, you will be encouraged to take a closer look. Don't panic! Say something like 'Well, perhaps I should examine the film more closely' and proceed more cautiously.

\section{Type II case: area of pathol- ogy obvious but diagnosis not immediately apparent}

- Give a full description of positive and relevant negative findings as concisely as possible.

- Remember control views when 
dealing with a contrast examination. Rather than stubbornly insisting on these immediately, ask 'May I assume that the control radiograph was normal?' The examiner must either give a truthful reply or provide the control radiograph.

- If you are confident that no further abnormality is present, then say, 'The rest of the film appears to be normal'.

- If you have not yet examined the entire film say 'I would just like to examine the rest of the film' and begin doing so. If there is more to see the examiner will usually remain silent. If detailed scrutiny of the rest of the film would only waste time this will usually be indicated, thereby providing an opportunity to summarise findings thus far and consider further steps toward diagnosis, e.g. 'The patient has a solitary pulmonary nodule and my considerations would by $\mathrm{X}, \mathrm{Y}$ or perhaps $\mathrm{Z}$.

- Do not feel obliged to make a diagnosis on a single film or study. If additional views or investigations such as tomography, CT or a barium swallow are indicated then justify the request by saying something like 'I am considering P, Q or possibly R. A chest CT may show fat or calcifications in the nodule or hilar adenopathy?'

- Note that expected findings should be volunteered before the next study is at hand, whenever possible.

- If you seem unsure, the examiner will often ask what investigation you think should be performed next. Be logical. Generally one should be sequential, i.e. special views, tomograms, contrast study, sonar, CT and then MRI, but this is only a broad guideline. For example, if you are dealing with musculoskeletal or CNS pathology, don't be afraid to suggest CT or MRI immediately.

- Remember the great mimickers: TB and lymphoma.

- AIDS will remain popular even if a cure is found.

- Additional clinical information can occasionally shed more light than further studies. Interstitial lung disease is such an example where clinical background can be more useful than, say, HRCT.

Here are examples of helpful clinical information: $(i)$ in the case of interstitial lung disease, enquire about duration of complaints (acute or chronic), immune status, dust/allergen exposure, drug history, known collagen-vascular disease or malignancy and involvement of other organ systems; (ii) in the case of bone lesions, enquire about the age of the patient, similar lesions elsewhere, a known primary malignancy or metabolic disturbance; (iii) the patient's name can indicate ethnicity. In certain cases, e.g. Gaucher's or sickle-cell disease, this can be helpful; and (iv) the skin, eyes, bones and GIT may be involved in diseases such as sarcoidosis and the sero-negative arthropathies.

- By now a definite diagnosis or at least a short differential should hopefully be possible. Present your argument by saying 'Based on the history and findings, I would consider $\mathrm{X}, \mathrm{Y}$ or possibly Z'. If the correct diagnosis doesn't feature in your selection, you will be asked about further possibilities. If it does, the examiner will possibly prompt you to select the single most likely aetiology from your list.

- If all has gone as planned, the films will be taken down and the next case will be given. If a case has gotten the better of you and you are wasting precious time, simply say 'Mr Examiner, I can't seem to get further on this case. May we please proceed with the next?'

\section{Type III case: an apparently 'normal' film}

- Remember that something is wrong! The chances of being presented with a normal film are very small indeed, but such urban legends do exist.

- After your initial 10 - 20 second scout, say 'At first glance I see no obvious abnormality so I am going to examine the film systematically', and do so.

- Enlightened examiners may steer you to the region or system you should concentrate on, saving time. Many will not.

- Bear in mind the history you were given. Very often it will hold a clue! Enquire about additional clinical aspects which could help.

- Never forget the so-called 'hidden' or 'review' areas, especially on chest films.

- Don't request additional views/ studies unless you have earned them by finding some possible abnormality or inadequately demonstrated region on the initial film which requires further scrutiny.

- It may be fatal to pronounce a film normal when something important cannot be seen or is obscured - for example $\mathrm{T} 1$ on a lateral neck. Beware also of bits hidden under film clamps. 
- If a classical clinical picture is painted, a helpful trick is to base an assumed diagnosis on the information you have been given and say 'Based on the history, I am looking for signs of XYZ'. A good example is pulmonary embolism.

- When you finally spot the abnormality the case becomes Type I or II.

- If after all this you still see nothing abnormal it is best to say so: 'Mr Examiner, I'm sorry but I cannot seem to find fault on this film. In so doing you are placing the ball in his court and he should guide you. The assumption being that you haven't missed some crucial finding such as free peritoneal air. A serious oversight!

\section{Some helpful 'don'ts'}

- Don't argue with an examiner, even if you know you are right.

- Don't list rare or obscure conditions at the top of a differential. The chances of being shown a case of Pelizaeus-Merzbacher disease are probably nil!

- Don't offer a differential list a mile long, in the hope your 'shotgun' approach may hit on the correct one.

- Don't ignore an examiner's input in favour of your own opinion. He's trying to help you.

- Don't insist on additional clinical information or studies when you are obviously overlooking something on the film at hand.

- Don't forget: common things occur commonly and hence if you suggest them, you will most commonly be correct.

- Don't listen to anything the previous candidate has to say. Examiners have plenty of films. And lastly, but not least

- Don't lose your cool. Remain calm under fire. An adrenaline overdose is no substitute for a clear head.

Please note that these 'classifications' I have offered are intended to present a different perspective on examinations and to guide possible problem approaches. They represent a condensation of my own observations and experiences as well as gems of wisdom conferred by tutors and colleagues along the way. The idea is definitely not to classify and categorise every film and approach each one recipestyle. Radiologists are neither librarians nor chefs and radiology can never be simple taxonomy or Boef Bourguignon! No magical recipe exists for guaranteed success. Ultimately every radiologist must develop his or her own unique problem-solving method. The common denominators in each approach will always remain knowledge, experience and technique, for which there are no substitutes.

All that remains is good luck, may the Force be with you and remember: this is only the beginning. In practice, every case is an exam case.

\section{In memoriam}

I would like to dedicate this piece to my friend and mentor Dr Tobie Rossouw, who passed away tragically and unexpectedly on 12 August 2001. His brilliant diagnostic mind and tireless dedication to our profession earned him great respect among colleagues and clinicians alike. The heartfelt sympathies of the radiology community go out to his wife Lona. 